\title{
Leibniz and the Metaphysics of Motion
}

\section{Edward Slowik}

\begin{abstract}
:
This essay develops a interpretation of Leibniz' theory of motion that strives to integrate his metaphysics of force with his doctrine of the equivalence of hypotheses, but which also supports a realist, as opposed to a fully idealist, interpretation of his natural philosophy. Overall, the modern approaches to Leibniz' physics that rely on a fixed spacetime backdrop, classical mechanical constructions, or absolute speed, will be revealed as deficient, whereas a more adequate interpretation will be advanced that draws inspiration from an invariantist conception of reality and recent non-classical theories of physics.
\end{abstract}


Among recent trends in the history and philosophy of science, the attempts to interpret Leibniz' physics using the conceptual resources employed by contemporary theories of classical physics have achieved little, if any, success. In response to the obvious difficulties associated with reconciling his alleged commitment to relational motion with a conservation law that is quite inhospitable to relational motion, commentators have espoused various strategies for circumventing this obstacle, such as the use of reference frames in a spacetime structure congenial to relational motion, or the endorsement of absolute motion or speed, as Leibniz' preferred view. ${ }^{1}$ Nearly all of the modern reconstructions, furthermore, attempt to reconstruct Leibniz' physics within the context of classical mechanics or classical gravitation theories, namely, by comparing Leibniz' conception of body, velocity, force, etc., against their modern usage, and then striving to find an equivalent notion within classical mechanics or gravitation theories that can represent Leibniz' usage. ${ }^{2}$

In this essay, these classical physics based reconstructions of Leibniz' physics will be evaluated and ultimately deemed inadequate, a verdict that is based not only on the evidence of the texts, but also due to the insufficiency of their results. Instead, an interpretation will be offered that, besides gaining textual support, draws upon a more

\footnotetext{
${ }^{1}$ For the spacetime approach to Leibniz, see: John Earman, World Enough and SpaceTime, Cambridge: MIT Press, 1989; Edward Slowik, "The 'Dynamics' of Leibnizian Relationism: Reference Frames and Force in Leibniz's Plenum”, Studies in History and Philosophy of Modern Physics 37 (2006), pp. 617-634. For the absolute speed interpretation, see: John Cook, “A Reappraisal of Leibniz's Views on Space, Time, and Motion”, Philosophical Investigations 2 (1979), pp. 22-63; John Roberts, "Leibniz on Force and Absolute Motion", Philosophy of Science 70 (2003), pp. 553-573.

${ }^{2}$ For example, see: Richard Westfall, Force in Newton's Physics, New York: Elsevier, 1971; Howard Bernstein, "Leibniz and Huygens on the 'Relativity' of Motion", Studia Leibnitiana 13 (1984), pp. 85-101; Richard Arthur, "Space and Relativity in Newton and Leibniz”, British Journal for the Philosophy of Science 45 (1994), pp. 219-240.
} 
metaphysical line of argument that bears a closer resemblance to non-classical theories of modern physics, such as the recent quantum gravity hypotheses (which attempt to connect quantum mechanics to General Relativity). In short, the only route to a successful construal of Leibniz' theory of motion must pass through his metaphysics of substance, a metaphysics which is inhospitable to the concepts of classical physics: specifically, any interpretation that downplays the metaphysical aspects of Leibniz' theory for a construction based entirely on concepts and strategies imported from classical mechanics or the modern spacetime approach associated with classical mechanics or gravitation theories, such as the idea of absolute motion, is thus inadequate. Some commentators have also questioned the adequacy of a spacetime reconstruction Leibnizian motion, it should be noted, yet no substitute interpretation has been offered so far. ${ }^{3}$ This essay will try to lay the groundwork for meeting this challenge: a reconstruction of Leibniz' theory will be advanced that places the emphasis on a fairly literal reading of his thesis dubbed, the "equivalence of hypotheses", a thesis that seems to infer that there are many different, but equal, hypotheses of motion, and hence different states of motion that can be assigned to a given body from the perspective of these different theories. Overall, it will be argued that the phenomena of motion led Leibniz to endorse a view that allowed a host of divergent hypotheses to remain consistent with our experience of motion, a view that is both hierarchical and perspectivalist in orientation, but which is grounded in an invariant feature, force, at a deeper level of ontology incompatible with classical physics. The interpretation advanced in this essay, therefore, neither saddles Leibniz with a crude form of idealism or

\footnotetext{
${ }^{3}$ See, Nicholas Huggett, "Can Spacetime Help Settle any Issues in Modern Philosophy?", philsci-archive.pitt.edu/id/eprint/3085 [accessed 2006].
} 
immaterialism, i.e., that only incorporeal beings (God, souls, etc.) and their mental content exist, nor does it equate with an objectivity-denying brand of subjectivism or relativism about material phenomena. In short, a realism concerning the external world is consistent with the "invariantist" strategy pursued in this essay.

After introducing the details of Leibniz' theory of motion, section 1 will offer an interpretation of his doctrine known as the equivalence of hypotheses while demonstrating the inadequacy of recent interpretations that rely on classical physics, namely, the absolute speed hypothesis. Our alternative interpretation of Leibniz' theory, which relies on a unique invariantist view of physical theories that differs significantly from the approaches derived from classical physics, will then be outlined in section 2.

\section{Leibnizian Motion and its Discontents.}

\subsection{Overview of Leibnizian Motion and the Equivalence of Hypotheses. One of the}

most familiar appearances of the dilemma can be found in the Discourse on Metaphysics $(1686):^{4}$

[I]f we consider only what motion contains precisely and formally, that is, change of place, motion is not something entirely real, and when several bodies change position among themselves, it is not possible to determine, merely from a consideration of

\footnotetext{
${ }^{4}$ The following abbreviations will refer to primary texts. [C]: Opuscules et fragments inédits de Leibniz, ed. by Louis Couturat, Paris, 1903; [A]: Sämtliche Schriften und Briefe, ed. by Akademie der Wissenschaften der DDR, Darmstadt and Berlin: AkademieVerlag, 1923. Cited with series, volume, and page; [GM]: Leibnizens mathematische schriften, ed. by Karl Gerhardt, Hildesheim: Olms, 1962. Cited with volume, page; [G]: Die philosophischen schriften von Leibniz, ed. by Karl Gerhardt, C. I. Hildesheim: Olms, 1965. Cited with volume, page; [L]: Leibniz: philosophical letters and papers, 2nd ed., ed. and trans. by Leroy Loemker, Dordrecht: Kluwer, 1969; [AT]: Oeuvres de Descartes, ed. by Charles Adams and Paul Tannery, Paris: J. Vrin, 1976. Cited with volume, page; [AG]: Leibniz: philosophical essays, ed. and trans. by Roger Ariew and Daniel Garber, Indianapolis: Hackett, 1989; [LoC]: The Labyrinth of the Continuum: Writings of 1672 to 1686, ed. and trans. by Richard Arthur, New Haven: Yale University Press, 2001.
} 
these changes, to which body we should attribute motion or rest, .... But the force or proximate cause of these changes is something more real, and there is sufficient basis to attribute it to one body more than another. Also, it is only in this way that we can know to which body the motion belongs. ${ }^{5}$

The first half of this quotation would seem to fit nicely with a strict relationist account of motion, for, as Leibniz puts it, "it is not possible to determine, merely from a consideration of these changes [in position], to which body we should attribute motion or rest". Nevertheless, in the second half of the above quotation, Leibniz goes on to assign individual motions to these bodies by means of their force: "there is sufficient basis to attribute it [force] to one body more than another", and "it is only in this way that we can know to which body the motion belongs". In his later Specimen Dynamicum (1695), he refers to these force-based motions as "true" motions: "[F]orce is something absolutely real in substances, even in created substances, while space, time, and motion are, to a certain extent, beings of reason, and are true or real, not per se, but only to the extent that they involve either the divine attributes (immensity, eternity, and the ability to carry out works), or the force in created substances". ${ }^{6}$ In the modern parlance, motion conceived "kinematically" (without considerations of force), is apparent or geometric motion, whereas motion conceived "dynamically" (under the action of forces) is true or real motion. Yet, by declaring that there is a difference between true and apparent motion, Leibniz' view parts company with relational motion, since a strict account of relational motion cannot admit any such distinction.

A further impediment for a relationist construal of Leibnizian motion concerns his conservation principle, $m v^{2}$ (roughly, the size of the body times the square of its

\footnotetext{
${ }^{5}$ G IV 444; AG 51.

${ }^{6}$ GM VI 247; AG 130-131.
} 
velocity). ${ }^{7}$ Since this law necessitates a series of privileged reference frames or perspectives for measuring this conserved quantity, namely, inertial (non-accelerating) reference frames, not all attributions of motion and rest to the bodies that undergo a relative change in position will uphold $m v^{2}$. An accelerating reference point or frame will likely attribute motions to these bodies that fail to conserve $m v^{2}$, and so it would appear that the assignments of motion or rest are not entirely arbitrary, contra the strict relationist view of motion. In the parlance of spacetime theories, the dilemma that Leibniz' physics presents for modern reconstructions is that his dynamical symmetries, i.e., the perspectives that preserve his conservation law, do not match his spacetime symmetries, i.e., the perspectives allowed for determining motion per se. A "NeoNewtonian" (or Galilean) spacetime, which possess an inertial structure that can delineate accelerating and non-accelerating motions, is the minimal structure required to uphold $m v^{2}$, since it is only in inertial frames that the conservation of $m v^{2}$ can be guaranteed. But, Leibniz' insistence that, in the absence of force, all assignments of motion are possible would seem to side with the so-called "Leibnizian" spacetime, which lacks inertial structure and thus cannot distinguish accelerating and non-accelerating motions. ${ }^{8}$ The spacetime theorist must therefore attempt to steer a course between the Scylla of too much structure (Neo-Newtonian spacetime), which violates strict relational motion by picking out preferred trajectories through space, and the Charybdis of too little structure

\footnotetext{
${ }^{7}$ See, e.g., GM VI 244-245; AG 128.

${ }^{8}$ See, Earman 1989, chap. 2, for the canonical presentation of these spacetime structures.
} 
(Leibnizian spacetime), whose inability to distinguish inertial paths undermines the application of the conservation law. ${ }^{9}$

Finally, the dichotomy between true and apparent motion would likely not be as puzzling if it were not for an additional doctrine known as the "equivalence of hypothesis" (hereafter, EH). The significance of EH for Leibniz' overall natural philosophy has long been a source of debate, for it seems to involve both the arbitrariness of the assignments of motion given a geometrical change of position, as discussed above, in addition to what is often referred to as the "Principle of Galilean Relativity", the hypothesis that the laws of physics are the same in all inertial frames (i.e., whether the frame is at rest or moving rectilinearly with a uniform speed):

[W]e must hold that however many bodies might be in motion, one cannot infer from the phenomena which of them really has absolute and determinate motion or rest. ... From this follows something that Descartes did not notice, that the equivalence of hypotheses is not changed even by the collision of bodies with one another, and thus, the laws of motion must be fixed in such a way that the relative nature of motion is preserved, so that one cannot tell, on the basis of the phenomena resulting from a collision, where there had been rest or determinate motion in an absolute sense before the collision. ${ }^{10}$

Leaving aside the possible conflation of relational motion and Galilean Relativity, perhaps the most controversial aspect of EH is that Leibniz appears to sanction something akin to a pragmatic or instrumentalist account of the truths associated with the individual states of bodily motion, a pragmatism that stems from a free choice of the approach or "point of view" adopted to describe the phenomena. More carefully, in the 1689 text, "On Copernicanism and the Relativity of Motion", Leibniz insists that the determination of

\footnotetext{
${ }^{9}$ See, Slowik 2006, for an examination of several reconstructions along these lines, although all of these reconstructions are ultimately deemed unsuccessful for the general reasons just outlined.

${ }^{10}$ GM VI 247; AG 131.
} 
the "greater intelligibility and simplicity" of an hypothesis, Ptolemaic and Copernican, is relative to a point of view, respectively, spherical astronomy and planetary theory—but, since the Ptolemaic and the Copernican hypothesis can assign different states of motion to the same body, such as the earth, it would appear to follow that the truths of an individual body's state of motion are thus relative to the pragmatic or instrumentalist consideration to adopt either spherical astronomy or planetary theory as one's point of view of material phenomena. After recounting the relative nature of motion and the equivalence of hypotheses, in the same manner as the quote offered above, he goes on to address the question of bodily motions:

But since, nevertheless, people do assign motion and rest to bodies, ... we must look into the sense in which they do this, so that we don't judge that they have spoken falsely. And on this matter we must reply that one should choose the more intelligible hypotheses, and that the truth of a hypothesis is nothing but its intelligibility. Now, from a different point of view, not with respect to people and their opinions, but with respect to the very things we need to deal with, one hypothesis might be more intelligible than another and more appropriate for a given purpose. And so, from different points of view, the one might be true and the other false. Thus, for a hypothesis to be true is just for it to be properly used.... [T] he truth of a hypothesis should be taken to be nothing but its greater intelligibility, indeed, that it cannot be taken to be anything else, so that henceforth there would be no more distinction between those who prefer the Copernican system as the hypothesis more in agreement with the intellect, and those who defend it as the truth. For the nature of the matter is that the two claims are identical; nor should one look for a greater or a different truth here. $^{11}$

In addition, Leibniz seems to rank the points of view according to their overall intelligibility, for while he regards "the Ptolemaic account is the truest one in spherical geometry, on the other hand the Copernican account is the truest theory, that is, the most

${ }^{11}$ C 590-591; AG 91-92. 
intelligible theory and the only one capable of an explanation sufficient for a person of sound reason". ${ }^{12}$

On the whole, it is difficult to gauge the precise scope and intended function of the Leibniz' "truth = most intelligent hypothesis" gambit. As argued by Lodge, since there are gradations of truth, it is more likely that Leibniz equates intelligible hypotheses with empirical adequacy, and not literal truth. ${ }^{13}$ For instance, if the Copernican hypothesis is the truest account, then some bodies would be judged to be at rest from the perspective of this hypothesis (such as a body or particle situated at the center of gravity of the solar system). Yet, Leibniz often claims that no body is entirely at rest, thus, to avoid contradiction, the empirical adequacy of an hypothesis seems the most plausible interpretation of his use of the term "truth" in this context. ${ }^{14}$ A passage that supports this interpretation comes from a 1694 letter to Huygens: "I hold, of course, that all hypotheses are equivalent, and when I assign certain motions to bodies, I do not and cannot have any reason other than the simplicity of the hypothesis, since I believe that one can hold the simplest hypothesis (everything considered) as the true one". ${ }^{15}$

1.2. Motion: Absolute or Hierarchical? Among the strategies for interpreting Leibniz' complex hypotheses of motion, a recent approach that has garnered some attention is to accept his distinction between true versus apparent motion, but without requiring an absolute space to ground true motion. Dubbed "absolute speed" by Roberts, the idea is that, given a change in position among several bodies (apparent motion), some of these

\footnotetext{
${ }^{12}$ C 591; AG 92.

${ }^{13}$ Paul Lodge, "Leibniz on Relativity and the Motion of Bodies", Philosophical Topics 31 (2003), p. 300.

${ }^{14}$ For the denial of complete rest, see, e.g., G VII 404; L 706.

${ }^{15}$ GM II 184-85; AG 308.
} 
bodies possess a real, force-induced motion (true motion) that is responsible for that observed change in position (hence true motion $=$ absolute speed $/$ motion). However, since absolute speed (hereafter, AS) does not entail absolute space, the AS interpretation does not violate Leibniz' long standing antipathy to absolute space. ${ }^{16}$ Unfortunately, AS runs counter to the evidence of the texts, which regularly dismisses the concept of absolute motion: for example, in the "On Copernicanism" tract, Leibniz states that "since space without matter is something imaginary, motion, in all mathematical rigor, is nothing but a change in the positions of bodies with respect to one another, and so, motion is not something absolute but consists in a relation". ${ }^{17}$ and, in more detail, he adds:

Since we have already proved through geometrical demonstrations the equivalence of all hypotheses with respect to the motions of any bodies whatsoever, however numerous, moved only by collisions with other bodies, it follows that not even an angel could determine with mathematical rigor which of the many bodies of that sort is at rest, and which is the center of motion for the others. And whether the others are moving freely or colliding with one another, it is a wondrous law of nature that no eye, wherever in matter it might be placed, has a sure criterion for telling from the phenomena where there is motion, how much motion there is, and of what sort it is, or even whether God moves everything around it, or whether he moves that very eye itself. $^{18}$

Given his repeated denials of absolute motion, and his above claim that EH is akin to an invariable law of nature that applies to collisions as well as planetary motions, the tenability of the AS hypothesis is thus called into question. ${ }^{19}$

In response to these worries, Roberts attempts to separate Leibniz' endorsement of EH from his various references to true motion:

${ }^{16}$ See Roberts 2003 for an elaborate treatment of AS, but it was earlier put forward by Cook 1979, and probably several others.

${ }^{17} \mathrm{C}$ 590; AG 91.

${ }^{18} \mathrm{C} 590$; AG 91.

${ }^{19}$ For denials of absolute speed, see, e.g., A.VI.iv.1968; GM VI 251. 
What, then, are we to make of the opening passage of "On Copernicanism" ... ? There, as we have seen, Leibniz argues that all frames of reference are equivalent for the purpose of describing the motions of bodies, and that we are justified in using the reference frame that makes the phenomena out to be most intelligible. But very early in this paper, Leibniz makes it explicit that in this context, by "motion" he means nothing more than "change of place" [AG 91]. As we have seen, Leibniz makes it clear, in other writings spanning the time of publication of "On Copernicanism", that this is not the only way of regarding motion. In the essay on Copernicanism, Leibniz is working with a relatively thin conception of motion by his own standards, and what he says there shouldn't be taken to trump what he says elsewhere about motion in the fullest sense. ${ }^{20}$

In short, Roberts claims that $\mathrm{EH}$ is restricted to only a "thin" kinematical/geometric conception of motion, whereas true motion, and hence absolute speed, constitutes a different account ("motion in the fullest sense"). Yet, Leibniz specifically mentions bodily collisions in the context of EH in the passage that Roberts' cites from "On Copernicanism" ("moved only by collisions with other bodies"), and it is these types of bodily interactions that serve as the basis for the introduction of the "true motion" concept in the Discourse and Specimen Dynamicum.

In fact, as persuasively argued by Ric Arthur, the assignment of true motion based on the notion of intelligibility, and thus $\mathrm{EH}$, is a common theme in much of Leibniz' mature work. ${ }^{21}$ A discussion of the motion of a body in a fluid, for example, prompts the following conclusion: "[a]nd granted that motion is a relative thing, nonetheless that hypothesis which attributes motion to the solid, and from this deduces the waves in the liquid, is infinitely simpler then the others, and for this reason the solid is adjudged to be the cause of the motion. Causes are not derived from a real influence, but from the

\footnotetext{
${ }^{20}$ Roberts 2003, pp. 559-560.

${ }^{21}$ Richard Arthur, "Leibniz on the Relativity of Motion", presentation, $6{ }^{\text {th }}$ Annual Conference of the Leibniz Society of North America, Montreal, 2012.
} 
providing of a reason". ${ }^{22}$ Since causes are obtained by finding a reason, i.e., the most intelligible hypothesis, and not through the transmission of a force, this demonstrates that EH applies to the types of phenomena covered in the Discourse, the work most often cited for introducing the true motion distinction. Leibniz' rationale for employing $\mathrm{EH}$ in these cases ultimately stems from his conception of substance, a conception which denies any interaction or causal influx between substances since each substance contain an expression of the entire world as an internal feature: "no real influence of [substances] on one another is intelligible. For whatever happens to each of them would flow forth from its own nature and notion even if all of the others were imagined to be absent, since each one expresses the world". ${ }^{23}$

Moreover, Leibniz' interpretation of bodily interactions, wherein the grounding substances as well as bodies do not really causally interact, plays a key role in his rejection of absolute motion. ${ }^{24}$ The non-interaction of bodies, he claims, provides the only explanation for the "phenomena of motion", for "every single body must be supposed to have a motion in common with any other, as if they were in the same ship, as well as its own motion, reciprocal to its bulk; how this could be so could not be imagined if motions were absolute and each body did not express all others" (c. 1686; A.VI.iv.317;

${ }^{22}$ A Specimen of Discoveries, c. 1686; A.VI.iv.1620; LoC 311; see also the AG 308 reference above, where greater simplicity is included within $\mathrm{EH}$, and would thus seem to be a form of greater intelligibility.

${ }^{23}$ A.VI.iv.1620; LoC 311.

${ }^{24}$ In trying to bridge the divide between non-interacting substances and material bodies, Leibniz often appeals to elasticity as a means of modeling the simple substance's internal expression of the world. He reasons that "no impetus is transferred from one body to another, but each body moves by an innate force, which is determined only on the occasion of, i.e. with respect to, another. For . . . the cause of the impulse one body gets from another is the body's elasticity itself, by means of which it recoils from the other" (A.VI.iv.1620; LoC 311). 
LoC 335). ${ }^{25}$ Importantly, this passage equates absolute motion with a motion that does not express all of the other bodily motions in the world, i.e., that is independent, thereby violating the pre-established harmony idea that informs his metaphysics. In an early essay, Leibniz make a similar point, commenting that "if motion is an affection, its subject will not be any one individual body, but the whole world. Hence all its effects must also necessarily be relative. The absolute motion we imagine to ourselves, however, is nothing but an affection of our soul while we consider ourselves or other things as immobile, since we are able to understand everything more easily when these things are considered as immobile". ${ }^{26}$

Finally, it worth quoting a longer discussion from the New System of Nature (1695), for it brings together Leibniz' conception of substance, true motions, and $\mathrm{EH}$, all within the context of the bodily interactions that make up his force-based physics:

For we can say that the substance, whose disposition accounts for change intelligibly, in the sense that we may judge that the other substances have been accommodated to this one in this regard from the beginning, according to the order of God's decree, is the substance we must consequently conceive as acting upon the others. Furthermore, the action of one substance on another is neither the emission nor the transplanting of an entity, as commonly conceived, and can reasonably be taken only in the manner just stated. It is true that we readily conceive emissions and receptions of parts in matter, by which we reasonably explain all of the phenomena of physics mechanically. But since material mass is not a substance, it is clear that action with respect to substance itself can only be as I have just described. . . . And as for absolute motion, nothing can fix it with mathematical rigor, since everything terminates in relations. This makes for the perfect equivalence of hypotheses, as in astronomy, so that no matter how many bodies we take, we may arbitrarily assign rest or a particular degree of speed to any body we choose, without being refuted by the phenomena of rectilinear, circular, or composite motion. However, it is reasonable to attribute some true motions to bodies, in accordance with the assumption that accounts for the

\footnotetext{
${ }^{25}$ Feb. 1677; A.VI.iv.1970; LoC 229.

${ }^{26}$ In this early discussion, Leibniz may have accepted the notion of a world body or motion, an idea that would ultimately be replaced by an infinity of simple substances in pre-established harmony. Either way, absolute motion is judged to violate his underlying metaphysics.
} 
phenomena in the most intelligible way, this denomination being in conformity with the notion of action we have just established. ${ }^{27}$

After denying that bodies communicate motion with one another, and denying absolute motion, he asserts that the most intelligible account of the true motions of bodies stems from the "notion of action". More carefully, the concept of action, from the first part of the passage, involves substances in pre-established harmony, and he concludes by adding that the most intelligible hypotheses of explaining the phenomena of bodily motion is "in conformity" with his theory of the action of (non-interacting) substances. In this context, the phrase, "the assumption that accounts for the phenomena in the most intelligible way", probably refers to the practical ability of the mechanical approach to determine the true motions of bodies (from the apparent) via his conserved quantity, $m v^{2}$, and the prior history of the system's motions and relationships. Returning to the earlier example (LoC 311), the decision to regard the solid as in motion, and not the liquid, would be the most intelligible way of explaining the phenomena (i.e., simplest), with the force of the moving body "in conformity" with his underlying metaphysics of non-interacting substances. To sum up, Leibniz' use of EH in this passage is based on the idea that the true motions that we assign to bodies resembles or mirrors the action of substances, even though substances do not truly interact, and bodies and their motions are not truly real.

Consequently, given the abundant evidence of the texts, Leibniz' account of true motion is, in fact, an instance of his EH doctrine, and not an alternative or contrary conception of motion. Since the phrase, "most intelligible way", stands as yet another instance of Leibniz' pragmatist approach to scientific explanation, it follows that the assignment of true motion is simply the best means of describing bodily phenomena from

${ }^{27}$ G IV 487; AG 145 . 
the perspective of the mechanical hypothesis - but there are many other hypotheses of motion. As argued in the "On Copernicanism" text, "one hypothesis might be more intelligible than another and more appropriate for a given purpose. And so, from different points of view, the one might be true and the other false" ${ }^{28}$ Accordingly, if we desire to designate individual bodily motions from the perspective of the mechanical hypothesis and the conservation of $m v^{2}$, then we can appeal to the activity of the substances that ground those material bodies as a deciding factor; however, that assignment of individual bodily motions does not entail that motion is something real or absolute, i.e., as real as substance and primitive force, or that the mechanical account of motion based on $m v^{2}$ is the one true account of bodily motion, and that all other accounts of bodily motion, such as the Tychonic, are false. The fact that the mechanical assessment of bodily motions based on $m v^{2}$ falls under EH is crucial in this respect, since it demonstrates that there is, so to speak, a hierarchy of hypotheses of motion constructed for different purposes. Presumably, the mechanical hypothesis is the most intelligible of all, since it more closely reflects, or is in conformity with, the action of simple substances, but this "hierarchical" conception of Leibniz' metaphysics of motion, as we will call it, runs counter to the AS hypothesis. Specifically, the AS hypothesis holds that true motions are absolute and that EH applies only to motion conceived as kinematic/geometric, hence the motions determined from the perspective of the mechanical hypothesis are metaphysically privileged, i.e., really true, whereas the motions determined from the Ptolemaic point of view are really false. But, as demonstrated above, the textual evidence points instead towards a different conception, a metaphysics wherein all of these different

${ }^{28}$ C 590-591; AG 91. 
hypotheses of motion fall under $\mathrm{EH}$ in a sort of hierarchical series that includes both the mechanical hypothesis and the purely kinematic.

Defenders of the AS hypothesis, moreover, construe the complexities associated with Leibnizian motion as stemming from the denial of absolute space rather than absolute motion: "I've argued that Leibniz's doctrine of force commits him to the doctrine of absolute speed, that absolute speed doesn't entail absolute space" ${ }^{29}$ Yet, besides the denial of absolute space, Leibniz repeatedly rejects absolute motion, and one of the rationales offered is that absolute motion does not "not express" all of the other bodies and their motions. The AS hypothesis not only fails to account for this aspect of Leibniz' system, but a body's absolute motion would, in fact, appear to be independent of all the other bodies and their motions, contra Leibniz' demand. For these reasons, an interpretation that is more consistent with the textual evidence is required.

\section{Realism and Leibnizian Motion.}

2.1. Realism versus Idealism. Under an idealist or immaterialist interpretation of Leibniz' theory, the instrumentalism or pragmatism that is seemingly embodied in EH is perfectly feasible, since only incorporeal beings (God, monads) and their perceptions exist. ${ }^{30}$ On the other hand, a realist interpretation of Leibniz' theory, which posits the existence of a mind-independent material world of some sort, confronts a much more difficult task given the instrumentalism inherent to $\mathrm{EH}$. A realist interpretation of Leibniz' theory, it should be noted, can accept the fact that the true nature of corporeal

\footnotetext{
${ }^{29}$ Roberts 2003, 569.

${ }^{30}$ For an idealist reading of this type, see, Montgomery Furth, "Monadology", Philosophical Review 76 (1967), pp. 169-200.
} 
existence is not identical to our mathematical and geometrical conceptions of that reality, or, to put it differently, that there is a degree of idealism in our mathematical grasp of matter. As Leibniz frequently comments, the continuous, holistic structures employed by mathematics are ideal, whereas matter is ultimately composed of discrete elements that aggregate to form macroscopic bodies. ${ }^{31}$ Nevertheless, even if bodies do not resemble our mathematical notions precisely, a realist interpretation must accept the existence of a material world apart from our minds/souls - to deny this inference is to abandon realism for some version of idealism. ${ }^{32}$

Yet, how can one reconcile a pragmatic approach to bodily motions, as exemplified in the hierarchical conception of EH defended in section 1, with a realist conception of physics, even granting this concession to the idealistic component latent in our mathematical conceptions? In particular, if one adopts the thesis that the assignment of individual bodily motions are relative to the overall theory used to explain the phenomena, such as the Tychonic, Ptolemaic, or Copernican theories-and that none are actually true in the literal sense (and the other false) — has one simply abandoned any plausible realist interpretation for a full-blown instance of idealism (since, e.g., the Tychonic and Copernican theories posit different states of motion to the earth)?

${ }^{31}$ See, e.g., G II 379. The mathematical basis of all of the hypotheses of motion, whether Ptolemaic, mechanical, etc., is clearly one of the motivating factors behind $\mathrm{EH}$, although the exact nature of the relationship between mathematics and reality is topic beyond the bounds of this essay. For example, in explaining how force can single out true motion (in the Leibniz-Clarke correspondence), Leibniz claims that "there is not any one body that is perfectly and entirely at rest, but we will frame an abstract notion of rest by considering the thing mathematically" (G VII 404; L 706). Hence, while no body is actually at rest, mathematical hypotheses can still admit that notion.

${ }^{32}$ Leibniz does, in fact, reject Berkeley-style idealism. For the anti-idealist case, see, e.g., Glenn Hartz, Leibniz's Final System, London: Routledge, 2007, pp. 81-82; and, Donald Rutherford, "Leibniz as Idealist", Oxford Studies in Early Modern Philosophy 4 (2008), pp. 141-190. 
The dilemma imposed by Leibniz' handling of EH can be surmounted by the realist if one is willing to accept a more nuanced and complex conception of his ontology than the standard classical physics-based strategies put forward thus far. The chief problem with the approach to Leibniz' physics based on theories of classical physics, such as classical mechanics or spacetime theories, is that they rely, not surprisingly, on the conceptions of space, time, and matter associated with classical mechanics or spacetime theories, but with the additional proviso that these structures do not support absolute space. While denying absolute space entails that these reconstructions are faithful to Leibniz' rejection of the so-called static and kinematic shift arguments in the Leibniz-Clarke correspondence - i.e., the possibility that the entire world could have, respectively, a different position in space or a different velocity — an ideal space alone does little to resolve the $\mathrm{EH}$ puzzle. ${ }^{33}$ By substituting an ideal space for a real space, but retaining the remainder of the classical mechanical ontology centered upon a realist construal of bodies and their motions, one remains mired in the non-realist consequences of EH surveyed above, namely, that individual states of motion become relative to the chosen theory.

The best strategy to deal with the EH quandary, therefore, is to make all of the geometrical aspects of the ontology of bodies, including both motion and bodily extension, mind-dependent to some degree, and thereby accommodate the subjective or instrumentalist implications of EH via a corresponding subjective or instrumentalist form of ontology as regards those geometrical features. This interpretation of Leibniz' material world, where space, time, bodies, and motion are all phenomenal or ideal to a varying extent, has the added advantage that it is, in fact, Leibniz' oft-repeated view. In the

\footnotetext{
${ }^{33}$ See, G VII 363-364; AG 325, for the shift arguments.
} 
Discourse, he argues that it is "possible to demonstrate that the notions of size, shape, and motion are not as distinct as imagined and that they contain something imaginary and relative to our perception, as do (though to a greater extent) color, heat, and other similar qualities, qualities about which one can doubt whether they are truly found in the nature of things outside ourselves". ${ }^{34}$ In essence, Leibniz rejects a sharp primary/secondary property distinction, placing the so-called primary properties, such as shape, size, and motion, alongside the secondary properties, like color and heat. This view, which Garber dubs “primary-quality phenomenalism", is related to, but more general than, Leibniz' belief (mentioned above) that our mathematical notions do not accurately correspond to material existents. ${ }^{35}$ By reckoning that bodily extension and motion "contain something imaginary and relative to our perception", one can thus consistently uphold the subjective element in EH since the individual motions of bodies can now be included within the category of the perceptually imaginary and relative. So, given this perceptual relativity as regards all of the geometrical aspects of bodies, the determination of the individual states of motion can be left to a pragmatic choice of theory. This interpretation avoids idealism or immaterialism, moreover, by accepting the existence of the material realm, although this material realm can only be objectively characterized in terms of force: it is the geometrization of force, by means of bodies, motions, etc., that is ideal and subjective, but not force itself - and so there is more to the world than just immaterial beings, God and monads, and their perceptions.

\footnotetext{
${ }^{34}$ G IV 437; AG 44; see also, A6.4.1504; L 365.

${ }^{35}$ Daniel Garber, Leibniz: Body, Substance, Monad, Oxford: Oxford University Press, 2009, p. 297.
} 
Taking this interpretational route, however, means abandoning any strategy based on a realist reading of classical mechanics or spacetime structures, since these realist strategies cannot allow their structures, nor the bodies within these constructions, to be relative or subjective in a way similar to the relativity of the perception of motion. The same holds for the absolute speed interpretation, which relies on Neo-Newtonian spacetime structure. ${ }^{36}$ If bodies and their accompanying spacetime structures are themselves relative or pragmatic, then these classical mechanical and spacetime strategies fail to provide a realist groundwork to resolve the EH puzzle.

What one requires, consequently, is an invariant or absolute ontological item at a level of reality deeper than classical mechanics and classical spacetime structures, an ontology that, while different from classical physics, can consistently explain the relative perspectives at the ontological level of bodies and motions normally associated with

\footnotetext{
${ }^{36}$ See, Roberts 2003. As regards the Special and General Theories of Relativity, allowing the shape (but not volume, rest mass, etc.) of bodies to be perspectival in the same fashion as the judgments of velocity (but not acceleration) is a consequence of its fourdimensional Minkowskian spacetime structure and its all-important invariant, the spacetime interval (Lorentzian metric). The many attempts by various Neo-Kantians and Logical Positivists to see Leibniz as presaging Mach and Einstein can be traced in part to Leibniz assertions on EH. Briefly, these twentieth century thinkers viewed Leibniz' EH as supporting the type of relational theory of motion championed by Mach, as well as Einstein's own early belief (later rejected) that General Relativity upholds Machian relationism. For some history, see, Howard Bernstein, "Leibniz and Huygens on the 'Relativity' of Motion”, Studia Leibnitiana 13 (1984), pp. 85-101; Vincenzo De Risi, "Leibniz on Relativity: The Debate between Hans Reichenbach and Dietrich Mahnke on Leibniz's Theory of Motion and Time", in Ralf Krömer and Yannick Chin-Drian (eds.), New Essays in Leibniz Reception: Science and Philosophy of Science 1800-2000, Basel: Springer, pp. 143-185, 2012. De Risi's investigation of EH may draw conclusions similar to those put forward in this essay, moreover, although he approaches these issues from the phenomenalist side in Leibniz' thought (whereas our study has strived to remain within the ontological): in particular, De Risi seems to deny that absolute motion applies to the noumenal (real) world (p. 163), which is one of the major conclusions advanced in this essay.
} 
classical physics — and, once again, this is exactly the strategy that Leibniz offers: "We must realize, above all, that force is something absolutely real in substances, even in created substances, while space, time, and motion are, to a certain extent, beings of reason, and are true or real, not per se, but only to the extent that they involve either the divine attributes (immensity, eternity, and the ability to carry out works), or the force in created substances [monads]". ${ }^{37}$ This invariant force is, of course, the primitive force of monads, but it is manifest at the bodily level as derivative force, the force measured by $m v^{2}$ from the mechanical perspective.

Although a full examination is beyond the bounds of this essay, a brief discussion is required concerning the nature of Leibnizian force and its relationship with $m v^{2}$. First, the force that brings about motion is not located at the level of his simple substances, or monads, since there is no space at this level: "there is no spatial or absolute nearness or distance between monads". ${ }^{38}$ Instead, monads possess primitive force, and primitive force manifests itself at the level of well-founded phenomena (i.e., the bodily level) as derivative force: a monad is "endowed with primitive power" such that the "derivative forces [of bodies] are only modifications and resultants of the primitive forces". ${ }^{39}$ Yet, it is the derivative forces that are associated with motion: "I, however, do not consider motion to be a derivative force but think rather that motion, being change, follows from such force" ${ }^{40}$ If derivative forces are, as it were, a determinate value of the primitive forces, and motion "follows from" derivative force, then it would seem natural to conclude that the forces that are implicated in motion, i.e., $m v^{2}$, also exist at the

\footnotetext{
${ }^{37}$ GM VI 247; AG 130.

${ }^{38}$ G II 451; L 604.

${ }^{39}$ G II 251; AG 176.

${ }^{40}$ G II 262; L 533.
} 
phenomenal level of bodies. Moreover, whereas monads are non-spatial, derivative force is coupled to the phenomena of extended bodies, which are aggregates of monads, or secondary matter: "the derivative force of being acted upon later shows itself to different degrees in secondary matter". ${ }^{41}$ Consequently, both bodily extension and the derivative force that is associated with motion, $m v^{2}$, are brought about by primitive force. Since Leibniz frequently characterizes derivative force as a limitation or modification of primitive force, an interpretation that deems both bodily extension and motion as perspectival in nature thus gains support. As he explains to De Volder, "unless there is some active principle in us, there cannot be derivative forces and actions in us, since everything accidental or changeable ought to be a modification of something essential or perpetual, nor can it contain anything more positive than that which it modifies, since every modification is only a limitation, shape a limitation of that which is varied, and derivative force a limitation of that which brings about the variation". ${ }^{42}$ Finally, from within the mechanical perspective based the on conservation law of $m v^{2}$, the only constraint on the perspectival assessments of bodily extension and motion is that they combine to uphold $m v^{2}$, with the invariance of that quantity reflecting the absoluteness of primitive force at the deeper monadic/substance level.

Unlike Descartes, who appears to have taken for granted that the application of his laws of motion are not undermined by the relativity involved in the assessment of motion (), Leibniz builds the relativity of motion directly into his overall ontology, with $m v^{2}$ representing, in an indirect and partial manner, an invariant feature that underlies all of the conflicting estimations of the bodily motions that constitute the mechanically-

${ }^{41}$ GM VI 236; AG 120.

42 June 30, 1704; G II 268-270; AG 179-180. 
conceived system. Consequently, despite their similarities on a purely mathematical basis, Descartes' quantity of motion and Leibniz' $m v^{2}$ operate in vastly different ways. ${ }^{43}$ The reciprocal nature of motion (i.e., the transfer between a body and its containing bodies) and his conservation law for the quantity of motion (roughly, size times speed) are, for Descartes, two separate issues that are only accidentally, and problematically, conjoined in his physics. Leibniz treats the relativity of the motion and its attendant conservation law, in contrast, as two interrelated effects of a deeper cause, force, whose reality is only indirectly and incompletely expressed through the quantity $m v^{2}$ employed by the mechanical perspective. A quick rationale for this strategy stems from his belief that force is real, whereas the geometrical properties of bodies are ideal; but, since $m v^{2}$ concerns those geometrical features, it automatically follows that it cannot reveal the full or real nature of force. This is consistent with Leibniz' explanation that motion is linked to derivative force, but that derivative force is an instantiation of the ontologically deeper "absolute" primitive force. ${ }^{44}$

At the level of material bodies, on the other hand, it is true that Leibniz hit upon a key discovery, represented by EH, that conjoins his conserved physical quantity with a group of spatiotemporal symmetries (i.e., the symmetries encoded within Neo-Newtonian spacetime and its inertial structure, a framework that constitutes the modern spacetime setting for modeling the Galilean relativity implicit in EH). But, any interpretation of

${ }^{43}$ On Descartes, see, Part II of the Principles, AT VIIIA 40-79.

${ }^{44}$ It is outside the bounds of this essay to examine the full range of interpretations of Leibnizian force and motion, their historical development in Leibniz' work and in the seventeenth century, or indeed to examine other aspects of his complex metaphysics. Rather, given limitations of space, the goal is to show how Leibnizian force in his mature period (1680s onward) can fit the invariantist interpretation of Leibniz' physics that will be outlined in section 2.2. 
Leibnizian force that limits its ontological scope to the material plane of bodies and motions fails to grasp its true significance. A case in point are the modern physics-based reconstructions of Leibniz' theory that, presumably, treat his conception of force and $m v^{2}$ as the components of a larger equation: "force $=m v^{2}$ ". From this framework, Leibniz' distinction between an invariant (non-relative) force and relative motion leads to serious difficulties, since force is the product of the square of velocity and the size of the body; so, if motion is relative, then force is relative. Russell states that this objection is "unavoidable on any relational theory of space", and Earman expresses the same misgivings. ${ }^{45}$ The answer to this conundrum, also argued by Lodge, is to jettison the

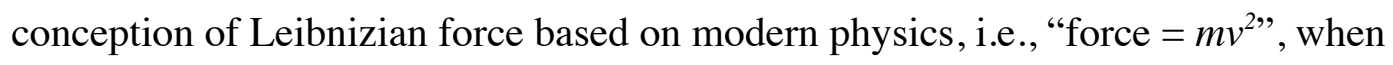
interpreting Leibniz' ontological use of that term, and the textual evidence supports this maneuver, as argued above. ${ }^{46}$

2.2. Outlining a Leibnizian Invariantist Ontology. In the recent literature on the metaphysics of objectivity and invariance, Leibniz' theory could stand as an early instance of what Debs and Redhead call "invariantism". ${ }^{47}$ In general, an invariantist reckons that objectivity is best understood as a feature of the world that remains fixed under changes in perspective, and incorporates both (i) inter-subjectivity, a purely epistemological feature that many observers share identically, and (ii) ontological independence, whereby it is stipulated that the observed invariant feature actually stems

\footnotetext{
${ }^{45}$ Betrand Russell, A Critical Exposition of the Philosophy of Leibniz, $2^{\text {nd }}$ ed., London: Routledge, 1992, p. 101; Earman 1989, p. 132.

${ }^{46}$ Lodge 2003, pp. 286-287.

${ }^{47}$ Talal Debs and Michael Redhead, Objectivity, Invariance, and Convention: Symmetry in Physical Science, Cambridge, MA: Harvard University Press, 2007, p. 61. Their work is influenced in part by: Hermann Weyl, Symmetry, Princeton: Princeton University Press, 1982; Robert Nozick, Invariances: The Structure of the Objective World, New York: Belknap, 2001.
} 
from a deeper ontological item that can exist apart from all observers (i.e., it is not a completely mind-dependent phenomena). By distinguishing the epistemological and ontological aspects of the invariance concept, this interpretation also provides a convenient means of separating a Furth-inspired fully idealist reading of Leibnizian force, which corresponds with (i), from a realist construal which must incorporate both (i) and (ii). In other words, the demand for inter-subjectivity is consistent with an idealist or immaterialist ontology that only accepts the existence of immaterial entities, whereas the stipulation that there is an ontological feature that exists in the external world, and which is responsible for the perspectival variance in our ascriptions of the individual states of motion, supports a corresponding materialist or physicalist inference (since force lies on the materialist side of the material/immaterial divide). Among the contemporary interpretations of Leibniz, Earman sides with route (i), contending that Leibniz' later ontology includes "only monads and their perceptions", wherein "the physical world is reduced to such perceptions". ${ }^{48}$ Whether or not Earman favors a purely immaterialist ontology is unclear, but most other commentators of Leibniz' physics and EH must include component (ii) alongside (i), since most of these commentators do not accept an idealist interpretation à la Furth. As discussed previously, most commentators of Leibnizian motion strive to locate a feature within his physics that can resolve the relational motion dilemma, such as the concept of absolute speed discussed in section 1.2, and this type of strategy only makes sense if one accepts the existence of the physical world along realist lines.

${ }^{48}$ Earman 1977, p. 223. Earman also claims that Leibniz "gives up a realist interpretation of space", and that "he did not pursue the middle path of constructing the physical world by assuming monads to have relative spatial relations", p. 226. 
Yet, if one does accept the invariantist interpretation Leibnizian force, and strives to synthesize both (i) and (ii), what are the ontological implications for his physics, as well as for his larger metaphysics? The strategy adopted by most commentators has been to interpret the quantity $m v^{2}$ as the invariant quantity that corresponds with Leibniz' conception of force, and then to employ the spatial translation symmetries intrinsic to Neo-Newtonian spacetime (see section 1.1) as the means of consistently measuring $m v^{2}{ }^{49}$ Unfortunately, spatial symmetries alone do not capture the type of ontology advanced in this essay, namely, where all of the properties of bodies, save force, are perspectival or relative to an instrumentalist or pragmatic choice of theory. As argued above, the classical mechanical or spacetime frameworks only allow changes in position or velocity (and shape, in the setting of Relativity theory), but the Leibnizian theory that we are considering allows all of the non-force aspects of bodies, such as volume, to be perspectival. Furthermore, since $m v^{2}$ is the invariant quantity from the perspective of the mechanical hypothesis, but other hypotheses of motion are guaranteed via $\mathrm{EH}$, the spacetime and classical mechanical approaches to Leibnizian invariantism are limited to just their perspective of the ontology of Leibnizian force, i.e., $m v^{2}$. Consequently, a different conception of the invariant associated with Leibnizian force is required that is more general than the standard conception based on $m v^{2}$.

Among modern hypotheses in physics, a closer match to the ontology of Leibniz' overall system would be the various "quantum gravity" proposals which posit physical processes that do not exist in space (spacetime) but which generate both matter and space at a higher level of reality. Like the monads and their primitive forces that bring about

\footnotetext{
${ }^{49}$ For example, Roberts 2003.
} 
matter and space in Leibniz' ontology, the "foundational" level physical processes put forward in many quantum gravity hypotheses are responsible for the emergence of both matter and space at the "secondary" material or bodily level, with the foundational and secondary levels possessing drastically different sets of properties. The quantities that might remain invariant in these quantum gravity theories, such as mass-energy, do not equate with Leibniz' $m v^{2}$, needless to say, but the general strategy is the same, namely, to explicate secondary level phenomena via invariant foundational level quantities and processes. It is beyond the bounds of this essay to examine these modern physical theories or render a verdict on the best analogues to Leibniz' view: the main point, in brief, is to demonstrate that the apparatus of spatial translations and symmetries utilized in classical mechanics and classical gravitational theories is not adequate to the task of explicating the ontology implicit in Leibniz' understanding of EH. ${ }^{50}$ Leibniz' theory is much more radical than classical physics, and thus requires a different stock of physical theories as a basis of comparison. Indeed, Leibniz often describes the material level of well-founded phenomena as akin to a rainbow, and rainbows are not a feature of the world that can serve as a fundamental ontological item; rather, rainbows are the results of a deeper physical ontology. ${ }^{51}$ Similarly, the holographic principle that motivates much work in quantum gravity contends, roughly, that our familiar three-dimensional material world (secondary level) is akin to a hologram image of quantum information "projected" from the more fundamental realm of the processes and entities that comprise quantum gravity theories (foundational level). As Susskind describes some of this work, "the

\footnotetext{
${ }^{50}$ On quantum gravity theories and the historical ontology debate for space, see, Edward Slowik, "The Deep Metaphysics of Space", philsci-archive.pitt.edu/id/eprint/8924 [accessed 2011].

${ }^{51}$ On the rainbow analogies, see, e.g., G II 436.
} 
combination of quantum mechanics and gravity requires the three dimensional world to be an image of data that can be stored on a two dimensional projection much like a holographic image", 52

Leibniz' rainbow analogy, along with the relationship between the foundational and secondary levels of his ontology, also draws into the discussion questions that pertain to supervenience or emergence. In short, given a foundation in force at the level of monads ( simple substances), as well as our realist construal of his overall system, does the matter, and hence, motion, that arises at the secondary level of well-founded phenomena count as a form of supervenience or emergence? The answer to this question ultimately resides in the process by which matter "results" from monads, a term often used by Leibniz to describe this relationship: e.g., "properly speaking, matter is not composed of constitutive unities [monads], but results from them". ${ }^{33}$ The main idea behind Leibniz' "results" description would appear to be a type of grounding or dependence relationship, so that if one thing is posited (monad), a different thing follows (matter). As Rutherford comments, "[r] esulting is best interpreted as a relation of ontological determination". 54 All told, the relationship of ontological dependence between monads and matter might thus be captured via the concept of supervenience, which can be defined in simple terms as: a property $\mathrm{X}$ supervenes on a property $\mathrm{Y}$ if and only if $\mathrm{X}$-properties covary with $\mathrm{Y}$ properties; or, alternatively, there can be no difference in the X-property without a

\footnotetext{
${ }^{52}$ Leonard Susskind, "The World as a Hologram", Journal of Mathematical Physics 36 (1995), p. 6377.

${ }^{53}$ G II 268; AG 179.

${ }^{54}$ Donald Rutherford, Leibniz and the Rational Order of Nature, Cambridge: Cambridge University Press, 1995, p. pp. 221-222.
} 
difference in the Y-property. ${ }^{55}$ Consequently, since the geometrical aspects of body are straightforwardly dependent on monads in the manner required to satisfy the definition of supervenience, monads serve as the subvenient base $(\mathrm{Y})$, and matter as the supervening property (X). There have been previous supervenience-based interpretations of Leibniz' monadic system, it should be noted, but these past attempts do not address, or to take into account, the EH quandary and its instrumentalist consequences.$^{56}$ The invariantist conception of Leibnizian force advanced above, nonetheless, might offer a method of bringing the EH component of Leibniz' system into a larger supervenience interpretation.

As for the prospects of a Leibnizian metaphysics of emergence, however, the question is more uncertain and open to interpretation, especially given the elusive nature of emergence itself. If the intuitive notion of an emergent property is employed — as a novel or fundamental supervenient property that arises from the subvenient base but that is not strictly reducible to that base- then Leibniz' monadic metaphysics would not seem to support emergence. Leibniz often comments that derivative force does not "contain anything more positive than that which it modifies [i.e., primitive force]", and that bodies are a mere aggregate of real unities; hence, it is hard to justify any interpretation that would regard the phenomena of monads (i.e., derivative force, bodies, space and time) as somehow novel or fundamental features of his system that surface at a higher ontological level than the monads. ${ }^{57}$

\footnotetext{
${ }^{55}$ For supervenience in general, see, Brian McLaughlin, "Emergence and Supervenience", in Mark Bedau and Paul Humphreys (eds.), Emergence, Cambridge: MIT Press, pp. 81-98, 2008.

${ }^{56}$ For example, Jan Cover, "Relations and Reduction in Leibniz", Pacific Philosophical Quarterly 70 (1989), pp. 185-211.

${ }^{57}$ G II 270; AG 180. Nevertheless, in the late correspondence with Des Bosses, Leibniz' metaphysical musings that relate to the "substantial chain" raise the intriguing possibility
} 
To conclude, the invariantist interpretation that we have explored in this essay would seem consistent with a form of supervenience, but it might fall short of emergence. This conclusion, therefore, challenges any ontological appraisal of Leibniz' theory of matter and motion that depends solely on the conceptual resources of classical mechanics and classical spacetime constructions (including the AS strategy). In brief, a supervenience relationship between a fundamental level and a secondary material level plays no role in the type of impact mechanics usually associated with Leibniz' EH by commentators, i.e., where the bodies that conserve $m v^{2}$ are often envisioned as like billiard balls on a flat plane. Classical mechanics is all secondary, material level for physical interactions of this kind, and this demonstrates, once again, that the standard Newtonian-inspired physics of bodily interactions is not the proper framework by which to interpret Leibniz' ontology of matter, motion, and force.

Lastly, how does the instrumentalist account of the truth of hypotheses factor into this

of a legitimate emergent property. Although the extent to which Leibniz seriously entertains the substantial chain is open to debate, this new metaphysical item would seem to function as a non-ideal link among monads in a corporeal substance. A substantial chains is defined as "something substantial which is the subject of [the monads"] common predicates and modifications, that is, the subject of the predicates and modifications joining them together" (G II 518; AG 203; emphasis added). Like the relationship between monads and matter, "monads are not really ingredients of this thing [substantial chain] which is added [to the monads], but requisites for it" (G II 435; AG 198). Overall, the substantial chain appears responsible for bringing about real extension in bodies, i.e., not merely phenomenal, for he argues that if these unifying substances were "supernaturally to cease, the extension which belongs to [a] body will also cease", and "[w]hat will remain is only a phenomenal extension, grounded in the monads" (G II 435; AG 198). Leibniz also explains that "[r]eal continuity can arise only from a substantial chain" (G II 518; AG 203). The substantial chain, accordingly, is tantamount to real continuity or extension in corporeal substance, and, since there is apparently nothing like real continuity or extension in the monads that are the requisites of these chains, the substantial chain could thus be judged to qualify as an emergent property. 
story? Leibniz' example of a rainbow might be useful in this regard. ${ }^{58}$ The cause of our perception of a rainbow, rain drops and light, are real entities that exist in world, but the properties that we ascribe to rainbows are utterly different (e.g., the rainbow's color, continuity, position in space, etc.). Since the observed phenomena do not accurately represent the true nature of the grounding entities, one might conclude that there exists a certain amount of latitude in explaining the behavior of that observed phenomena. Specifically, Leibniz may have reasoned that, since none of the geometrical hypotheses that treat material-level bodily phenomena actually correspond to the true ontology at the foundational level of monads, it follows that an array of different explanatory hypotheses are consistent with the phenomena at the material level, and thus the only criterion for deciding among these competing hypotheses must be their simplicity from a particular standpoint (e.g., Ptolemaic in spherical geometry, etc.). In other words, if the secondary level of extended bodies is not the true ontology, but is closer to the phenomena of rainbows, then truth cannot be assigned to these hypotheses in any straightforward fashion, and so a different means of evaluation must be procured (such as instrumentalism).

${ }^{58}$ Such as G II 436. 